Published in final edited form as:

Curr Opin Ophthalmol. 2019 May ; 30(3): 166-178. doi:10.1097/ICU.0000000000000569.

\title{
Advances in Dry Eye Disease Treatment
}

\author{
Erin C. O'Neil, MD ${ }^{1}$, Matthew Henderson, BA ${ }^{1}$, Mina Massaro-Giordano, MD", Vatinee Y. \\ Bunya, MD ${ }^{1}$ \\ ${ }^{1}$ Scheie Eye Institute, University of Pennsylvania, Philadelphia, PA
}

\begin{abstract}
Purpose of review: The prevalence and burden of dry eye disease continues to grow at a rapid pace, creating an increased need for new therapies. In a sector once limited to only a handful of treatments, clinicians now have multiple options available for patients who fail traditional therapies. This review summarizes the various treatment options available to clinicians treating complex dry eye disease patients.
\end{abstract}

Recent findings: As we better-understand the multifactorial mechanisms leading to dry eye disease, treatments increasingly focus on the amelioration of the underlying deficiencies and inflammation, rather than on transient symptomatic relief alone. Most topical medications seek to replace deficient growth factors and/or decrease inflammation on the ocular surface. The majority of new devices and procedures seek to treat meibomian gland dysfunction, with one new device stimulating tear production through utilizing the nasolacrimal reflex pathway.

\section{Summary:}

Clinicians have more options at their disposal in the treatment of dry eye disease than ever before, including topical medications and devices.

\section{Keywords}

dry eye disease; evaporative dry eye; meibomian gland dysfunction; dry eye treatment

\section{Introduction}

Current studies estimate that approximately 16.4 million Americans (6.8\% of the adult US population) have been diagnosed with dry eye disease (DED). ${ }^{1}$ An additional 6 million (2.5\% of adult Americans) may experience dry eye symptoms but have not been diagnosed with DED, placing the total estimated burden of disease at $9.3 \%$ of adult Americans. The prevalence of DED is higher among women as compared to men and increases with age. ${ }^{1,2}$ One recent population based study found a prevalence of $11.3 \%$ among all adults greater than 50 years of age and as high as $22.8 \%$ among women greater than 75 years of age. ${ }^{1}$

Corresponding Author: Vatinee Bunya, MD, 51 N. $39^{\text {th }}$ Street, Philadelphia, PA 19104, Phone: (215) 662-9791, Fax: (215) 243-4695, vatinee.bunya@uphs.upenn.edu.

Conflicts of interest

MMG: Celularity (consultant); Glaxo-Smith Kline (consultant); PRN Omega (personal financial interest)

ECO, MH: None 
Based on this, we can anticipate that the prevalence of DED will continue to increase as the population ages. The burden of DED on affected patients is significant, with effects on visual function, activities of daily living, professional work, and quality of life. ${ }^{1,2}$ Direct costs from doctor's visits, medications, and procedures and indirect costs from decreased productivity, reduced quality of life, and general impairment in functioning make DED a substantial economic burden, as well. ${ }^{1-4}$ A recent economic analysis estimated that the average annual direct cost of managing an American dry-eye patient was $\$ 783$, which when adjusted for the prevalence of dry eye, amounted to $\$ 3.84$ billion across the U.S. healthcare system. 5

Systematic diagnosis and monitoring of dry eye disease can be achieved through the use of various validated measures of both signs and symptoms of disease. Commonly utilized subjective scales include the Ocular Surface Disease Index (OSDI) and the Symptom Assessment iN Dry Eye (SANDE). The OSDI is a 12-item questionnaire that evaluates symptoms over the preceding week. Scores range from 0-100 with higher scores indicating more severe disease. The SANDE utilizes a visual analog scale and asks patients to rate both severity and frequency of dry eye symptoms. ${ }^{6}$ These may be used in conjunction with clinical exam findings such as corneal and conjunctival staining with fluorescein and/or lissamine green, tear film break up time, and Schirmer testing. Point-of-care in-office testing can be helpful in supplementing the evaluation and may include the measurement of tear film osmolarity and MMP-9 testing. Meibography, or infrared imaging of the meibomian glands, can be helpful in evaluating the severity of meibomian gland dysfunction. Patients with moderate-to-severe disease will often require a combination of treatments aimed at targeting the inflammatory, evaporative, and aqueous deficient components of their disease. Individualized treatment regimens should focus on the underlying pathophysiology in order to optimize results.

Traditional dry eye treatments include over-the-counter artificial tears, warm compresses, and lid hygiene with baby shampoo. ${ }^{7}$ Topical cyclosporine A (CsA) $0.05 \%$, a fungal antimetabolite that has found use as an anti-inflammatory drug due to its ability to decrease IL-2 mediated T-cell activation, received FDA approval for dry eye disease in 2003 and has been in regular clinical use for the past 15 years. It is a common next line dry eye therapy for those who have failed the conservative measures above. An estimated $48.2 \%$ of chronic dry eye disease patients have filled a prescription for CsA, despite mixed physician opinion on the drug's efficacy. ${ }^{8}$ Multiple studies have found patients treated with CsA experience symptomatic improvement, lower Ocular Surface Disease Index (OSDI) scores, and improved Schirmer testing and tear film breakup time (TBUT) results. However, despite its long track record and frequent use, active questions still surround the use of topical CsA, including optimal concentration/dose, necessary length of treatment, and which patients are most likely to benefit from its use ${ }^{8-10}$ Furthermore, ocular burning is a common side effect of topical CsA (occurring in up to $17 \%$ of patients) and is the most common reason for discontinuing treatment by patients. ${ }^{1-14}$ Additional side effects include conjunctival hyperemia, discharge, epiphora, eye pain, foreign body sensation, pruritis, and visual disturbance such as blurry vision. ${ }^{11}$ In addition, the estimated cost of a 30-day supply of topical cyclosporine is $\$ 293.45$, making it the most expensive of the commonly prescribed ophthalmic medications, presenting a barrier to use by some patients. ${ }^{15}$ 


\section{Therapeutics}

\section{Medications}

Topical prescription medications discussed here aim to decrease inflammatory mediators and are therefore likely to be most effective in patients with evidence of a significant

inflammatory component to their disease, such as patients with moderate-to-severe aqueous tear deficiency (with or without Sjogren's syndrome), or graft-vs-host-disease.

Lifitegrast-In July 2016, lifitegrast 5\% became the second FDA approved topical ocular anti-inflammatory medication for the treatment of DED. The drug mimics the intercellular adhesion molecule-1 (ICAM-1), blocking interactions between ICAM-1 and lymphocyte functional associated antigen-1 (LFA-1), which are instrumental in T-cell activation and migration. ${ }^{16,17}$ Results of three phase III clinical trials (OPUS 1-3) demonstrated improvement in both signs and symptoms of dry eye disease. Notably, in a pooled analysis of the OPUS- 2 and OPUS- 3 studies, lifitegrast significantly improved the primary symptom outcome of change from baseline eye dryness score (EDS) (0 to 100 visual analogue scale [VAS]) score within 14 days and sustained this effect in a pooled analysis at the study's endpoints of 84 days (treatment effect 9.92 point decrease in EDS (VAS) score; 95\% CI 7.01-12.83; $p<0.0001) .{ }^{17}$ By comparison, topical cyclosporine typically takes 3 months to demonstrate clinical efficacy. ${ }^{17-19}$ The safety of lifitegrast was well demonstrated in the SONATA study, which followed 331 patients (220 lifitegrast vs 111 placebo) for one year and found no significant adverse events. In addition, plasma concentrations of lifitegrast remained low and no systemic immunosuppression or local opportunistic infections occurred..$^{20}$

Advantages of topical lifitegrast suggested by the above studies include relatively rapid effect, long-term safety, and improvement in both the signs and symptoms of dry eye disease. However, there are possible side effects including instillation-site irritation, instillation-site pain, instillation-site reaction, reduced visual acuity, and dysgeusia. ${ }^{17,20,21} \mathrm{In}$ addition, further study is needed to determine which patients respond best to lifitegrast, such as patients with known higher levels of inflammation such as patients with Sjogren's syndrome or graft-vs-host disease. It is also unknown whether concomitant use of lifitegrast with topical corticosteroids can result in faster symptomatic relief, as has been found in prior research on CsA use in combination with corticosteroids. ${ }^{17,22}$ Future studies regarding the effectiveness of the concomitant use of lifitegrast and cyclosporine are also needed.

\section{Blood products}

In recent years, platelet-derived products have found widespread use in wound healing and tissue regeneration. ${ }^{23}$ Application of these products in DED makes use of the numerous similarities between natural tears and serum, including lipid, proteins, antimicrobial elements, and growth factors. ${ }^{23,24}$ Autologous and/or allogenic serum drops have been shown across multiple studies to improve both symptoms and objective signs of dry eye disease. ${ }^{25-28}$ Most recently, interest has grown in the use of other blood-derived products, specifically eye-platelet rich plasma (E-PRP) and plasma rich in growth factors (PGRF). These newer products contain three to five times the platelet concentration compared to 
serum drops and aim to maximize the concentration of growth factors, cell adhesion molecules and alpha-granule released cytokines. ${ }^{23}$ E-PRP and PGRF also do not contain leukocytes, which are present in serum drops and may increase levels of pro-inflammatory cytokines that are detrimental to the ocular surface. ${ }^{24}$ These higher concentrations of growth factors and other beneficial factors may result in substantial therapeutic effects on the ocular surface.

For example, Alio et al. studied 368 patients with moderate to severe dry eye treated with EPRP six times daily in a series of one to five consecutive rounds, each lasting six weeks (64.4\% of patients required only one round). After one round, $87.5 \%$ of patients reported subjective improvement, while $76.1 \%$ had improvement in corneal fluorescein staining and $28.8 \%$ had at least one line of gain in vision. ${ }^{29}$ In this study, some patients received up to five rounds of treatment with rare adverse events, suggesting long term use is likely safe, though formal studies are pending. However, despite some evidence of efficacy as treatments for DED, blood products are not FDA approved, so their access is limited and often costs are not covered by most insurances. ${ }^{30}$

\section{Procedures}

Available in-office procedures primarily target inspissated meibomian glands, using heat or mechanical energy to express glands and restore natural flow of meibum. As such, they are best suited for patients with a significant component of evaporative dry eye disease and meibomian gland dysfunction.

Intense pulsed light-Intense pulsed light (IPL) involves targeting pigmented or vascular lesions with visible and infrared light, which, upon absorption, is converted to destructive heat. Though used primarily in dermatology for its FDA-approved indication of treating telangiectasias, IPL has more recently been used as an off-label treatment for evaporative dry eye mediated by MGD, often in patients who also suffer from rosacea. ${ }^{31}$ IPL therapy involves direct application of $500 \mathrm{~nm}$ light to the skin, coagulating underlying blood vessels. Proposed mechanisms of efficacy include reduced inflammatory mediators and bacterial overgrowth by destruction of eyelid telangiectasias as well as melting of viscous meibum, allowing improved flow. ${ }^{31}$

In recent years, there has been increasing interest in the study of IPL for the treatment of DED. Toyos et al first described a treatment protocol for the use of IPL in dry eye disease in 2005. In their protocol, an IPL shield is placed over a patient's cleaned face. Cold ultrasound gel is applied to the treatment area, from tragus to tragus. Treatment settings range from 8 $\mathrm{J} / \mathrm{cm}^{2}$ to $20 \mathrm{~J} / \mathrm{cm}^{2}$, depending on disease severity. Two passes are performed. The gel is then removed and manual meibomian gland expression is performed using a cotton-tipped applicator on the palpebral conjunctiva and the physician's finger on the external lid $\operatorname{skin}^{31,32}$ (Figure 1).

In 2015, Toyos and colleagues published a retrospective review of 91 patients presenting with dry eye symptoms who were treated with IPL. They found that $93 \%$ of patients had improvement in symptoms and $87 \%$ improvement in TBUT over a median of four follow-up visits. Thirteen percent of patients reported adverse events, mostly related to localized 
redness and swelling, but there were no significant adverse events. ${ }^{31}$ In addition, several other retrospective studies published have also indicated improvement in signs and symptoms of dry eye disease following IPL therapy. ${ }^{33,34}$

Craig et al. published the first prospective results of IPL use for the treatment of MGD. ${ }^{35}$ In this double-masked, paired-eye, placebo-controlled study, 28 patients with mild to moderate MGD received three IPL treatment sessions to a randomly selected eye, while the other eye was assigned to serve as a sham-treated control. Treatment sessions were conducted at 1, 15, and 45 days from baseline and consisted of IPL treatment application to four periocular zones inferior to the eye. Lipid layer grade and noninvasive tear break-up time both improved significantly from baseline to day 45 in the treatment eye, but not in the control eye (both $\mathrm{p}<0.001$ ). Subjective self-reported dry eye symptoms using the VAS also significantly improved in the treated eye but not in the control eye $(\mathrm{p}=0.015)$. Tear evaporation rate and meniscus height, however, were not significantly different in the treated eye compared to the control eye. ${ }^{35}$

More recently, Dell et al. published prospective results that further demonstrated the therapeutic potential of IPL for MGD-mediated evaporative dry eye. ${ }^{36}$ They studied 80 eyes of 40 patients across two sites, providing four treatments three weeks apart, each of which included 10-15 pulses of IPL over the cheeks and nose, followed by manual meibomian gland expression of both upper and lower lids. Outcomes analyzed by change from baseline to final follow-up 15 weeks after initial treatment session. The number and severity of dry eye signs and symptoms including tear breakup time, meibomian gland score, corneal fluorescein staining, and SPEED questionnaire scores, all improved significantly ( $\mathrm{P}<0.001$ for all outcomes) from baseline to final follow-up. Tear film osmolarity improved by $-7 \%$ $(\mathrm{p}<0.005)$ in the 20 patients with abnormal results at baseline, but lipid layer thickness did not improve significantly. ${ }^{36}$

While these results are encouraging for the role of IPL in the treatment of dry eye disease, there are limitations to its use. For example, only lightly pigmented skin (Fitzpatrick Skin Types 1-4) is eligible for IPL treatment, since side effects such as hypopigmentation are more common in darker skin types. ${ }^{37}$ Upper eyelids are also not considered a safe target due to the risk of light absorption by pigmented intraocular structures, such as the iris. ${ }^{38}$ Other limitations include cost, as treatments are not covered by most insurances and maintenance treatments are often need annually or bi-annually. ${ }^{33}$

Vectored Thermal Pulsation (LipiFlow ${ }^{\mathrm{TM}}$ )—-LipiFlow ${ }^{\mathrm{TM}}$ (TearScience, Morrisville, $\mathrm{NC}$ ) is a device that combines meibomian gland expression with heat, in a technique referred to as vector thermal pulse therapy, for the in-office treatment of MGD. During this procedure the device applies heat over the palpebral conjunctiva of the upper and lower eyelids, while providing pulsatile external pressure (Figure 2).

Since Lane et al. first published positive results of LipiFlow ${ }^{\mathrm{TM}}$ use as compared to warm compresses in improving both signs and symptoms of DED in 2012, ${ }^{39}$ other studies have confirmed the benefits of LipiFlow ${ }^{\mathrm{TM}}$. Some studies have reported that a single treatment's effects lasting six to nine months or longer. ${ }^{40-43}$ However, an independent, randomized, 
masked, placebo-controlled clinical has not been conducted to provide strong evidence longterm benefits from a single treatment. Kim et al. published a retrospective review of 189 eyes that underwent thermal pulsation treatment. ${ }^{44}$ They reported that in 39 eyes with abnormal tear film osmolarity (defined as $>307 \mathrm{mOsms} / \mathrm{L}$ ), thermal pulsation treatment significantly improved values from an average of 317.1 to $306.6 \mathrm{mOsms} / \mathrm{L}$ ( $\mathrm{p}=0.002$ ). In the same study, MMP-9 assays were positive in $50 \%$ of 114 eyes pretreatment but only $26 \%$ of eyes post-treatment $(\mathrm{p}<0.0001) .{ }^{44}$ More recently, Hagen et al. published a prospective, randomized, parallel-group study comparing a single LipiFlow ${ }^{\mathrm{TM}}$ treatment to a 3-month course of doxycycline for the treatment of in 28 patients with MGD. ${ }^{45}$ Following treatment, SPEED scores improved in both groups but were significantly better in the LipiFlow ${ }^{\mathrm{TM}}$ group as compared to the doxycycline group (5.42 vs $9.42, \mathrm{p}=0.030$ ). The two groups were comparable in post-treatment meibomian gland function scores, TBUT, and corneal and conjunctival fluorescein staining. ${ }^{45}$ These results suggest that Lipiflow ${ }^{\mathrm{TM}}$ may be a preferred treatment for patients with meibomian gland dysfunction while avoiding the potential side effects and complications of long-term macrolide therapy.

Side effects for Lipiflow ${ }^{\mathrm{TM}}$ are minimal and include eye and eyelid discomfort that resolve soon after treatment, but there are limitations of this modality. ${ }^{46}$ The single-use, disposable applicators that comprise the ocular component of this device are expensive and not covered by most insurances, making Lipiflow ${ }^{\mathrm{TM}}$ potentially cost prohibitive. ${ }^{7}$ Despite evidence of its potential effectiveness in treating MGD, a randomized, double masked, placebo-controlled clinical trial is needed to further evaluate treatment duration.

Meibomian Gland Probing-In 2010, Maskin reported a novel probing technique for treating MGD using a beveled, $2 \mathrm{~mm}$ solid stainless steel probe directly into meibomian gland orifices. ${ }^{47} \mathrm{He}$ reported that 24 out of 25 patients had immediate relief in tenderness and all patients had persistent relief 4 weeks later. Repeat treatments were only required in 5 of the patients with an average follow-up of 11.5 months. ${ }^{47}$ In 2018, Maskin et al reported post-probing meibography results of 50 eyelids from 28 patients. The authors reported that $36 \%$ of lids appeared to have signs of individual gland growth with a mean follow-up time of 10 months, while detailed analysis of 13 lids demonstrated a $6.23 \%$ increase in mean individual gland area. The authors recognized not only lengthening of shortened glands but also partial restoration of faded glands and speculated that there was possible new gland growth. ${ }^{48}$ Also published in the last year, Incekalan et al. evaluated both effectiveness and speed of relief among 80 eyes of 20 patients treated with either conventional meibomian gland disease treatments (consisting of warm compresses, eyelid massage and cleansing, artificial tears, topical antibiotics, oral omega-3 supplementation, and oral azithromycin) or a combination of conventional treatments above with a single session of intraductal meibomian gland probing performed at first visit. ${ }^{49}$ Patients were followed for three months after the initial visit or procedure. OSDI, Schirmer testing, tear film breakup time, Oxford grading of ocular surface, and both meibum expressibility and quality improved in both groups, though the subgroup treated with probing had significantly faster improvement in all measurements. ${ }^{49}$

Several other authors have previously published on the efficacy of meibomian gland probing with encouraging results. ${ }^{50-54}$ Wladis reported on a series of 40 patients with average OSDI 
of 78.11, all with ocular and facial rosacea, treated with a single session of intraductal meibomian gland probing. Mean OSDI decreased to $37.54(\mathrm{p}<0.05)$ at one-month followup and 43.00 at six-month follow-up $(\mathrm{p}<0.05)$. Nine out of the ten patients previously on oral doxycycline were able to discontinue use. ${ }^{54}$ In a similar study by Sik Sarman et al. in 2016 of 58 eyes, which allowed for multiple treatments, mean OSDI score decreased from a mean of 79.7 at baseline to 22.9 at three months with an average of 1.7 sessions of probing. However, patients in this study were prescribed corticosteroids after the procedure and instructed to perform warm compresses and lid massage, which may have contributed to their superior results. ${ }^{53} \mathrm{Ma}$ and Lu compared intraductal meibomian gland probing with topical corticosteroids ( $0.1 \%$ fluorometholone) to use of fluorometholone alone in 49 patients. Patients who received intraductal meibomian gland probing had faster symptomatic relief (76\% at one day post-operative) as well as significantly greater improvement in ocular symptom scores and meibum scoring at one week and one month follow-up as compared to the steroid-only group. The group that underwent probing also experienced a significantly greater increase in TBUT than the steroids-only group $(8.2+/-2.1$ vs $7.0+/-3.0, \mathrm{p}=0.029)$. Both groups continued prior conservative treatments. Other measured parameters such as Schirmer I score were not significantly different between the two groups. ${ }^{51}$

In 2017, Syed and Sutula reported a new method of meibomian gland probing in which an operating microscope is used, von Grafe forceps are placed at the base of the tarsal plate and used to express meibum while a $2 \mathrm{~mm}$ followed by a $4 \mathrm{~mm}$ probe are used to break through obstructions and dilate the glands. $91.4 \%$ of treatments in their series of 70 eyelids resulted in symptomatic improvement, though more than half had relapse of symptoms during follow-up (range 1 to 64 weeks, mean 12.9 weeks). ${ }^{55}$

None of the papers we reviewed commented on patient discomfort as a disadvantage of this treatment method, but this remains a possible drawback to intraductal probing. The effectiveness of this procedure likely also depends on the skill and experience of the performing surgeon. Overall, more large-scale studies with long-term follow-up are needed to better assess the effectiveness of this treatment.

MiBoThermoflo-The Mibo Thermoflo (MiBo Medical Group, Dallas, TX) is an in-office therapeutic device for the treatment of MGD related DED, which aims to maximize meibum liquefaction and secretion, thus improving tear film quality (Figure 3). During the procedure, ultrasound gel is applied to the heated handheld probe, which is then used to gently massage the external lids for 8 to 12 minutes. ${ }^{56}$ The manufacturer recommends three treatments, each two weeks apart, and evaluating patients after to determine if they require future treatments. While no studies on its efficacy have been published yet, preliminary data from a trial sponsored by the manufacturer showed significant improvement in TBUT, osmolarity, SPEED, and OSDI four months after treatment in 51 patients. ${ }^{56}$ However, Kendrick \& Alloo reported in a case study, that the treatment was ineffective in raising the average palpebral conjunctiva temperature above $40^{\circ} \mathrm{C}$, the temperature required for effective meibum liquidification. ${ }^{57,58} \mathrm{~A}$ randomized, masked, placebo-control clinical trial still needs to be conducted to further investigate the treatments efficacy and safety.

Curr Opin Ophthalmol. Author manuscript; available in PMC 2020 May 01. 
BlephEx ${ }^{\mathrm{TM}}$-BlephEx $\underset{\text { TM }}{\mathrm{TM}}$ (Scope Ophthalmics, London, UK) is an in-office treatment for blepharitis to supplement at-home lid scrubs. This handheld device spins a disposable, single-use medical grade micro-sponge to remove debris from lashes and lids. In a preliminary prospective study, Connor et al. demonstrated the positive effects of BlephEx in treating dry eye symptoms; in the small study of 20 patients, the authors found that MGD and blepharitis grading (using Efron Grading Scale), TBUT, and OSDI improved significantly four weeks after BlephEx separately reported on a group 10 patients who tested positive for MMP-9 who were subsequently treated with Blephex treatment. ${ }^{60} \mathrm{~A}$ randomized, masked, placebo-control clinical trial is still needed to further investigate the treatments safety and efficacy compared to other blepharitis treatments.

TearCare ${ }^{\circledR}$ System-The TearCare ${ }^{\circledR}$ System (Sight Sciences, Menlo Park, CA) is an inoffice treatment slated to become available in 2019, which aims to combine the therapeutic effect of warm compress therapy with manual meibomian gland evacuation to treat MGD. The procedure involves placement of single-use, flexible $\mathrm{iLid}^{\mathrm{TM}}$ published clinical trial applicators over each tarsal plate which deliver constant, regulated heat at $41^{\circ} \mathrm{C}-45^{\circ} \mathrm{C}$ over a 12 minute treatment time, during which the patient is encouraged to blink normally to allow natural meibum expression (Figure 4). This is then followed by manual meibum expression by the physician immediately following removal of the iLid applicators. ${ }^{61}$ The manufacturer-sponsored, sole published clinical trial of the TearCare ${ }^{\circledR}$ System, in which 24 patients were randomized to receive a single treatment session with the TearCare® System followed by meibomian gland expression or daily warm compresses for five minutes over four weeks. At the four-week follow-up visit, mean TBUT increased by $11.7+/-2.6$ seconds in the TearCare group as opposed to a decrease of $0.3+/-1.1$ seconds in the warm compress group $(\mathrm{p}<0.001)$ with statistically significant results maintained over the six month follow-up period. TearCare ${ }^{\circledR}$ group patients also experienced statistically significant improvement in corneal and conjunctival staining as well as subjective symptoms as measured by the SPEED, OSDI, and SANDE questionnaires over the entire six-month follow-up period. ${ }^{61}$ The treatment was well tolerated and no adverse events were reported during the trial. Larger, randomized, masked, placebo-control clinical trials need to be conducted in the future to further evaluate the efficacy and safety of the TearCare ${ }^{\circledR}$ System. Data on patient comfort, treatment cost, duration of effect, and reliability of the heating of lids to the required $40^{\circ} \mathrm{C}$ temperature all remain to be published.

\section{Devices}

There are a variety of device options for the treatment of dry eye, each with unique indications. For example, lacrimal intranasal neurostimulation may be most useful in patients with aqueous tear deficiency whereas scleral lenses are of particular benefit in patients with an irregular corneal surface, neurotrophic disease, or graft-versus-host disease. These should be considered on an individualized basis for patients unresponsive to alternative therapeutic options.

Intranasal tear neurostimulation (TrueTear $\left.{ }^{\circledR}\right)$ - A new device that was FDAapproved for the treatment of DED is the Intranasal Tear Neurostimulator (ITN, TrueTear®, 
Allergan, plc) (Figure 5). The ITN was designed to stimulate the mucosal nerves via small electrical currents to increase natural tear production via the nasolacrimal reflex pathway of the lacrimal function unit. Briefly, the lacrimal function unit includes the lacrimal glands and the ocular surface, including the conjunctiva, cornea, and goblet cells, as well as the sensory and motor nerves that connect these structures to maintain tear production and quality. The afferent pathway of this system includes the ophthalmic and maxillary branches of the trigeminal nerve, with abundant nerve fiber endings in the nasal mucosa. ${ }^{62}$ Stimulation of these nerve fibers has been shown to increase tear production. Conversely, lack of stimulation of these nasal mucosal fibers of the trigeminal nerve, for instance in patients with trigeminal nerve ablation, reduced air flow due to allergy, or with tracheostomy, has been shown to result in decreased tear production. ${ }^{63}$ The device consists of two small probes, designed for one to be placed in each nostril, with five levels of stimulation intensity to be selected by the user.

In 2016, Friedman et al. published the results of a pilot study sponsored by Allergan-owned Oculeve of 40 subjects who were instructed to use the ITN device four times daily for 180 days. They found that mean Schirmer scores were significantly higher following nasal stimulation with the device than prior to stimulation and that corneal, conjunctival staining and ocular symptom scores (as measured by OSDI) were significantly reduced from baseline at day $180 .{ }^{64}$ Gumus et al. demonstrated that a single intranasal application of the ITN device increased tear meniscus height (measured via AS-OCT) by $28.8 \%$ in dry eye patients. ${ }^{65}$ Degranulated to non-degranulated goblet cell density (GCD) ratios in dry eye patients increased to $4.71 \pm 4.48$ when compared to baseline $(0.74 \pm 0.62)$ and to extranasal (0.57 \pm 0.54$)$ application (p-values $<0.001) .{ }^{65}$ In 2018, Sheppard et al. published results of two clinical trials of the ITN, both sponsored by Allergan; In the first, a single-day crossover study, patients underwent Schirmer testing before and after three test applications of the device: active intranasal, inactive intranasal (sham), and extranasal. Mean Schirmer scores were significantly greater with active intranasal $(25.3 \pm 1.5 \mathrm{~mm})$ vs extranasal $(9.5 \pm 1.2 \mathrm{~mm})$ and sham $(9.2 \pm 1.1 \mathrm{~mm})$ applications $(\mathrm{p}<0.001) .{ }^{66}$ In the second study, patients used the device 2-10 times daily for 180 days. At follow-up, stimulated Schirmer scores were significantly as compared to un-stimulated scores $(17.3 \pm 1.3 \mathrm{~mm}$ vs $7.9 \pm 0.7 \mathrm{~mm}, \mathrm{p}<0.0001){ }^{66}$

The most common side effects of the ITN device include nasal discomfort, burning, and pain, nosebleeds, transient electrical discomfort, nasal congestion, facial pain, and headaches. ${ }^{64-66}$ The device cannot be used in patients with cardiac pacemakers, implanted or wearable defibrillator, or other implanted metallic or electronic deice in the head or neck, a hypersensitivity to the hydrogel that coats the device probes, or chronic nosebleeds or other conditions that can cause increased bleeding. The cost of the device can also be limiting, as the device is roughly $\$ 1000$ and a month supply of disposable tips is approximately $\$ 50$, neither of which are covered by most insurance plans.

Scleral Lenses-Scleral lenses were previously used primarily for the treatment of corneal ectasias but more recently have been studied for use in ocular surface disorders. These lenses rest on the sclera, creating with a fluid-filled reservoir over the more sensitive cornea. There are several scleral lenses that are currently available that can be helpful in treating dry eye. 
Perhaps the most well known scleral lens used to treat ocular surface disease is the BostonSight ${ }^{\circledR}$ PROSE (Prosthetic Replacement of the Ocular Surface Ecosystem) lens (Boston Foundation for Sight, Needham, MA). The PROSE lens is custom designed using patient eye measurements so that the lens is not touching the apical or peripheral cornea but still maintaining minimal movement and optimizing fluid ventilation. An early retrospective review of 49 consecutive patients (76 eyes) with ocular surface disease treated with this lens found a $92 \%$ improvement in quality of life as a result of reduction in photophobia and pain. $53 \%$ of patients in this study achieved 2 or more Snellen lines of improvement in visual acuity. ${ }^{67}$ Agranat et al. reported long-term follow-up data and found that $64 \%$ of patients using PROSE for ocular surface disease reported continued wear at five years. In patients with OSD and continued wear at give years, National Eye Institute Visual Function Questionnaire (NEI VFQ-25) scores increased initially and after five years of wear (mean increase +27.17 and +18.80 , respectively). ${ }^{68}$ A number of other retrospective studies have further demonstrated the ability of PROSE to significantly improve dry eye signs and symptoms in OSD, including various cases that result from Stevens Johnson Sydrome, chronic GVHD, and even neurotrophic keratopathy following skull base tumor resection. ${ }^{69-75}$ In a prospective, interventional case series of 38 patients with ocular surface disease, Stason et al. reported that PROSE significantly improved visual acuity and visual functioning, according to the NEI VFQ-25. ${ }^{76}$

However, one major limitation to the use of scleral lenses for dry eye disease is the lengthy and costly fitting process that may not be covered by insurance. In addition, there are currently only 12 eye care institutions that offer PROSE treatment, which can further limit accessibility to patients due to proximity or the cost of travel. In light of these limitations, the Boston Foundation for Sight launched a new scleral lens fitting system in January 2017 called BostonSight ${ }^{\circledR}$ SCLERAL. This web-based, data-driven fitting system allows patients to be fitted with far fewer modifications and by their regular, local eye care practitioner, thus providing a less costly and more convenient alternative to the PROSE system, though less customized.

Another option for scleral lenses became available in 2013 to patients with unique anterior ocular surface anatomy in whom standard scleral lens fitting is unsuccessful. The EyePrintPro ${ }^{\mathrm{TM}}$ lens uses an imprint of the ocular surface and converts it into a 3D digital model, which is then printed as a lens. ${ }^{77}$ The resulting lens offers central and peripheral corneal clearance while landing tangential to the scleral surface while avoiding vasculature, even in the setting of abnormalities such as blebs, pterygia, pingueculae, and mucosal grafts. Nguyen et al. published a small retrospective review of 14 eyes of 10 patients fitted with EyePrint Prosthetics lenses and reported that all patients experienced improvement in dry eye symptoms redness, and pain. Six out of seven patients who previously wore other lenses reported significantly greater comfort with the EyePrintPro ${ }^{\mathrm{TM}}$ lens. ${ }^{77}$ However, this was a single small study and further larger studies are needed that include the assessment of ocular signs before and after use of lens. In addition, the cost of the lens may be prohibitively expensive for some patients.

Amniotic Membrane-Amniotic membranes have been used for the treatment of various ocular surface disorders, including limbal stem cell deficiency, infectious keratitis, corneal 
burns, persistent epithelial defects, and conjunctival reconstruction after symblepharon removal. Not only do they provide coverage of the epithelium to allow healing, but also impart anti-inflammatory properties. There are currently two commercially available varieties, either cryopreserved at $-80^{\circ} \mathrm{C}$, such as the ProKera ${ }^{\mathrm{TM}}$ (Bio-Tissue, Inc, Miami, FL), or sterilized dehydrated and stored at room temperature, such as the AmbioDisk ${ }^{\mathrm{TM}}$ (Katena Products, Inc, Denville, NJ).

ProKera $^{\mathrm{TM}}$ is available as a device that can be placed on a patient's eye in the office and has a ring that holds the amniotic membrane transplant (AMT) in place. The original ProKera ${ }^{\mathrm{TM}}$ is indicated for moderate to severe indications, including neurotrophic PED, severe infectious keratitis, post-PRK haze, and Salzmann nodular degeneration; however, Bio-tissue recently started offering the ProKera ${ }^{\mathrm{TM}}$ SLIM and ProKera ${ }^{\mathrm{TM}}$ PLUS. The ProKera ${ }^{\mathrm{TM}}$ SLIM is indicated for mild to moderate conditions, while the ProKera ${ }^{\mathrm{TM}}$ PLUS is recommended for severe indications that require longer treatment.

In 2016, Cheng et al. published a small retrospective review of 10 patients with dry eye disease treated with the ProKera ${ }^{\mathrm{TM}}$ Slim amniotic membrane. The patients achieved a statistically significant decrease in symptoms, use of topical medications, corneal staining, and conjunctival hyperemia. ${ }^{78}$ More recently, McDonald et al. reported on 97 eyes of 84 patients with severe dry eye disease despite maximum conventional therapies that underwent treatment with the ProKera ${ }^{\mathrm{TM}}$ Slim. Following treatment duration of 5.4 days, $88 \%$ had improved ocular surface. DEWS scores decreased from $3.25 \pm 0.5$ at baseline to $1.44 \pm 0.6$ at 1 week ( $p<0.001)$ with sustained result of $1.47 \pm 0.6$ at 3 months $(p<0.001)$ without any serious adverse events. ${ }^{79}$ With promising early results as above, further study is warranted to determine the duration of effect from membrane placement as well as comparison of the two currently available products.

\section{Pipeline}

Despite the considerable number of treatment options for dry eye disease, many patients fail to improve sufficiently with currently available treatments. Thus, the development of additional therapeutics is critical. We will briefly cover a few medications that have demonstrated promise in early clinical trials and could potentially be added to dry eye treatment regimens in the future.

Lacritin-Lacritin is an ocular specific glycoprotein secreted primarily by acinar cells of the lacrimal gland. Its primary role seems to be increasing basal tear secretion. ${ }^{80,81}$ Studies have shown that lacritin levels are significantly decreased in patients with Sjogren's syndrome as compared to healthy controls. ${ }^{81}$ In a murine model of SS dry eye disease, topical lacritin has been found to increase tear secretion, decrease lissamine green staining, and reduce signs of epithelial damage ${ }^{80}$ Phase I/II clinical trials of lacritin in patients with Primary Sjogren's disease are currently underway with estimated completion date of 2019.

Lubricin-Lubricin (PRG4) is a mucin-like glycoprotein that is expressed by the normal ocular surface and suppressed by inflammatory cytokines, including those implicated in dry eye disease. Lubricin's function is to decrease friction between the cornea and the conjunctiva and eyelid and it likely comprises an essential part of the ocular surface 
glycocalyx, preventing epithelial dysfunction and degradation. ${ }^{82,83}$ In 2017, Lambiase et al reported results of a two-week randomized, double blinded study comparing topical lubricin to topical sodium hyaluronate $0.18 \%$ in patients with moderate dry eye disease. Lubricin significantly outperformed sodium hyaluronate in ameliorating both signs and symptoms including foreign body sensation, blurry vision, photophobia, corneal staining, and tear film breakup time among others. ${ }^{83}$

Thymosin B4-Thymosin $\beta 4$ is a G-actin binding protein found in nearly all cells and in ocular surface fluid, which has been found to improve epithelial healing and down-regulate pro-inflammatory cytokines ${ }^{84,85}$. Early studies demonstrated its efficacy in neurotrophic keratitis and corneal wound healing. ${ }^{84,86,87}$ In 2015 , Sosne et al. first reported the use of topical thymosin beta4 (RGN-259) for the treatment of dry eye disease. In this phase 2, randomized, blinded, placebo-controlled trial, patients treated with RGN-259 for 28 days reported a $35.1 \%$ improvement in OSDI scores vs controls $(\mathrm{p}=0.0141)$ and $59.1 \%$ reduction in corneal staining as compared to controls $(\mathrm{p}=0.0108)$, though both groups did experience improvement. ${ }^{84}$ This study was followed in 2018 by a head-to-head comparison of RGN-259 vs. prescription agents including cyclosporine, lifitegrast, and diquafosol in a murine model. RGN-259 drops performed comparably or better than all prescription options in all parameters measured, which included increased tear production, improved corneal smoothness, decreased fluorescein staining, decreased epithelial detachment, and increased conjunctival goblet cell and mucin production. 88

Amniotic membrane extract eye drops-Much like cryopreserved amniotic membrane, amniotic membrane extract eye drops (AMEED) are proposed to impart a myriad of beneficial substances including growth factors, cytokines, and collagens to promote corneal wound healing, inhibit fibroblast activity, and decrease inflammation on and within the ocular surface. ${ }^{89}$ Amniotic membrane extract and AMEED have primarily been studied for use in patients with chemical burns or ocular injury rather than DED specifically. However, a variety of products are available without FDA regulation that are marketed for the treatment of DED and clinical trials are ongoing for use in patients with graft-vs-host disease and post-PRK dry eye. ${ }^{89}$ These drops are not covered by insurance so cost is expected to be prohibitive in many cases. The widespread use of AMEED may be limited by production, as manufacturing processes remain varied and challenging; processing and preserving may affect ultimate function. ${ }^{89}$

New Cyclosporine Formulations-With Restasis $®$ going off-patent, a number of pharmaceutical companies are in the process of developing novel cyclosporine A formulations. Eye drops with a higher concentration of CsA than Restasis ${ }^{\circledR}$ have been approved in Europe, Japan, and other countries. ${ }^{90-93}$ A Phase III clinical trial is currently underway for a Seciera ${ }^{\mathrm{TM}}$ (Sun Pharma, Mumbai, India), which has a CsA concentration almost twice that of Restasis ${ }^{\circledR}(0.09 \%$ vs. $0.05 \%)$. Phase II/III results, reported by Tauber et al. demonstrate an earlier onset of action in the $0.09 \%$ formulation compared to $0.05 \%$ and similar safety and tolerability profiles. ${ }^{94}$ Recently, Novaliq (Heidelberg, Germany) published Phase II results for $\mathrm{CyclASol}{ }^{\circledR}\left(0.1 \% \mathrm{CsA}\right.$ in EyeSol ${ }^{\circledR}$, water-free drug delivery solution) 
that suggested their formulation has an earlier onset of action than Restasis ${ }^{\circledR}$ and is safe and tolerable. ${ }^{95}$

In addition, compounded cyclosporine-based formulations are available for clinical use. For example, Klarity-C (Imprimis Pharmaceuticals, San Diego, California) is $0.1 \%$ CsA in a chondroitin sulfate emulsion. Chondroitin sulfate has been shown to be an effective lubricant, have anti-inflammatory properties of its own, and reduce corneal edema. ${ }^{96}$ However, large clinical trials are needed to evaluate the efficacy and safety of Klarity-C.

\title{
Conclusions
}

In recent years, there has been an enormous increase in research into new therapeutics for dry eye disease. There has been an increase in the development of therapies that target meibomian gland dysfunction and evaporative dry eye. The most notable shift appears to be a focus on identifying treatments that may reverse the underlying etiology, such as inflammation, rather than simply addressing symptoms by hydrating and lubricating the ocular surface. In particular, we note the use of biologic and biologically derived products such as blood serum and plasma, amniotic membranes and naturally occurring glycoproteins. Scleral lenses, such as the BostonSight SCLERAL lens and the EyePrintPro $^{\mathrm{TM}}$ lens, have also emerged as viable treatment options for ocular surface disease. Finally, the introduction of the intranasal neurostimulator represents a new approach to treatment of dry eye and may offer an alternative or supplement to traditional eye drop therapeutics. However, despite the increase in newly available treatments, there is a continued need for novel and effective treatment options. Fortunately, there is increased interest in the development of new therapeutics with several promising drugs currently undergoing clinical trials.

\section{Acknowledgments}

\author{
Financial support and sponsorship: \\ VYB: National Eye Institute R01 EY026972; Research to Prevent Blindness \\ ECO, MH, MMG: None \\ VYB: Celularity (consultant); Bausch \& Lomb/Immco Diagnostics (grant support)
}

\section{References:}

1. Farrand KF, Fridman M, Stillman IO, Schaumberg DA. Prevalence of Diagnosed Dry Eye Disease in the United States Among Adults Aged 18 Years and Older. Am J Ophthalmol 2017;182:90-98. [PubMed: 28705660]

2. Pflugfelder SC. Prevalence, burden, and pharmacoeconomics of dry eye disease. Am J Manag Care. 2008;14(3 Suppl):S102-106. [PubMed: 18452369]

3. Dalzell MD. Dry eye: prevalence, utilization, and economic implications. Manag Care. 2003;12(12 Suppl):9-13.

4. Patel VD, Watanabe JH, Strauss JA, Dubey AT. Work productivity loss in patients with dry eye disease: an online survey. Curr Med Res Opin 2011;27(5):1041-1048. [PubMed: 21417803]

5. Yu J, Asche CV, Fairchild CJ. The economic burden of dry eye disease in the United States: a decision tree analysis. Cornea 2011;30(4):379-387. [PubMed: 21045640]

Curr Opin Ophthalmol. Author manuscript; available in PMC 2020 May 01. 
6. Amparo F, Schaumberg DA, Dana R. Comparison of Two Questionnaires for Dry Eye Symptom Assessment: The Ocular Surface Disease Index and the Symptom Assessment in Dry Eye. Ophthalmology 2015;122(7):1498-503. [PubMed: 25863420]

7. Thode AR, Latkany RA. Current and Emerging Therapeutic Strategies for the Treatment of Meibomian Gland Dysfunction (MGD). Drugs. 2015;75(11):1177-1185. [PubMed: 26130187]

8. Deveney T, Asbell PA. Patient and physician perspectives on the use of cyclosporine ophthalmic emulsion $0.05 \%$ for the management of chronic dry eye. Clin Ophthalmol 2018;12:569-576. [PubMed: 29615833]

9. Sacchetti M, Mantelli F, Lambiase A, Mastropasqua A, Merlo D, Bonini S. Systematic review of randomised clinical trials on topical ciclosporin A for the treatment of dry eye disease. Br J Ophthalmol 2014;98(8):1016-1022. [PubMed: 24344232]

* 10. Rhee MK, Mah FS. Clinical utility of cyclosporine (CsA) ophthalmic emulsion $0.05 \%$ for symptomatic relief in people with chronic dry eye: a review of the literature. Clin Ophthalmol 2017;11:1157-1166. [PubMed: 28790800] This review of literature summarizes conflicting results regarding the efficacy of cyclosporine, particularly highlighting the challenges associated with correlating clinical signs with reported symptoms.

11. Allergan I. Restasis (cyclosporine ophthalmic emulsion) $0.05 \%$ full prescribing information. In: 2018.

12. Barber LD, Pflugfelder SC, Tauber J, Foulks GN. Phase III safety evaluation of cyclosporine $0.1 \%$ ophthalmic emulsion administered twice daily to dry eye disease patients for up to 3 years. Ophthalmology. 2005;112(10):1790-1794. [PubMed: 16102833]

13. Mah F, Milner M, Yiu S, Donnenfeld E, Conway TM, Hollander DA. PERSIST: Physician's Evaluation of Restasis((R)) Satisfaction in Second Trial of topical cyclosporine ophthalmic emulsion $0.05 \%$ for dry eye: a retrospective review. Clin Ophthalmol 2012;6:1971-1976. [PubMed: 23226002]

14. Perry HD, Solomon R, Donnenfeld ED, et al. Evaluation of topical cyclosporine for the treatment of dry eye disease. Arch Ophthalmol 2008;126(8):1046-1050. [PubMed: 18695097]

* 15. Newman-Casey PA, Woodward MA, Niziol LM, Lee PP, De Lott LB. Brand Medications and Medicare Part D: How Eye Care Providers' Prescribing Patterns Influence Costs. Ophthalmology. 2018;125(3):332-339. [PubMed: 28625684] Cross-sectional study of Medicare Part D 2013 prescriber public use files, reporting that eye care providers generate the highest percentage of brand name medication claims compared to all other specialties. Glaucoma medications account for $54 \%$ of total cost, the largest subspeciality. Dry eye disease is the second costliest category, driven primarily by cyclosporine, which accounts for $\$ 371$ million, or $17 \%$ of total cost among eye care providers.

* 16. Lollett IV, Galor A. Dry eye syndrome: developments and lifitegrast in perspective. Clin Ophthalmol 2018;12:125-139. [PubMed: 29391773] In this review article, the authors highlight the inflammatory cascades that contribute to dry eye disease while reviewing available treatments that target inflammation specifically: corticosteroids, cyclosporine, and lifitegrast.

** 17. Donnenfeld ED, Perry HD, Nattis AS, Rosenberg ED. Lifitegrast for the treatment of dry eye disease in adults. Expert Opin Pharmacother. 2017;18(14):1517-1524. [PubMed: 28841079] A review of the mechanism of action and pharmacokinetics of lifitegrast along with a summary of major clinical trials that led to FDA approval.

18. Sall K, Stevenson OD, Mundorf TK, Reis BL. Two multicenter, randomized studies of the efficacy and safety of cyclosporine ophthalmic emulsion in moderate to severe dry eye disease. CsA Phase 3 Study Group. Ophthalmology. 2000;107(4):631-639. [PubMed: 10768324]

19. Stevenson D, Tauber J, Reis BL. Efficacy and safety of cyclosporin A ophthalmic emulsion in the treatment of moderate-to-severe dry eye disease: a dose-ranging, randomized trial. The Cyclosporin A Phase 2 Study Group. Ophthalmology. 2000;107(5):967-974. [PubMed: 10811092]

20. Donnenfeld ED, Karpecki PM, Majmudar PA, et al. Safety of Lifitegrast Ophthalmic Solution 5.0\% in Patients With Dry Eye Disease: A 1-Year, Multicenter, Randomized, Placebo-Controlled Study. Cornea 2016;35(6):741-748. [PubMed: 27055211]

* 21. Holland EJ, Luchs J, Karpecki PM, et al. Lifitegrast for the Treatment of Dry Eye Disease: Results of a Phase III, Randomized, Double-Masked, Placebo-Controlled Trial (OPUS-3). Ophthalmology. 2017;124(1):53-60. [PubMed: 28079022] This phase III trail demonstrated 
significant symptomatic relief of dry eye disease in patients treated with lifitegrast as compared to placebo, as early as 14 days into treatment and sustained at 12 weeks.

22. Sheppard JD, Donnenfeld ED, Holland EJ, et al. Effect of loteprednol etabonate $0.5 \%$ on initiation of dry eye treatment with topical cyclosporine 0.05\%. Eye Contact Lens 2014;40(5):289-296. [PubMed: 25083776]

23. Drew VJ, Tseng CL, Seghatchian J, Burnouf T. Reflections on Dry Eye Syndrome Treatment: Therapeutic Role of Blood Products. Front Med (Lausanne). 2018;5:33. [PubMed: 29527528]

* 24. Anitua E, Muruzabal F, Tayebba A, et al. Autologous serum and plasma rich in growth factors in ophthalmology: preclinical and clinical studies. Acta Ophthalmol 2015;93(8):e605-614. [PubMed: 25832910] Review of factors within serum associated with wound healing and a summary of early data regarding the efficacy of serum derived medications in the treatment of ocular surface disease.

25. Celebi AR, Ulusoy C, Mirza GE. The efficacy of autologous serum eye drops for severe dry eye syndrome: a randomized double-blind crossover study. Graefes Arch Clin Exp Ophthalmol 2014;252(4):619-626. [PubMed: 24566903]

26. Hussain M, Shtein RM, Sugar A, et al. Long-term use of autologous serum $50 \%$ eye drops for the treatment of dry eye disease. Cornea 2014;33(12):1245-1251. [PubMed: 25299423]

27. Kojima T, Ishida R, Dogru M, et al. The effect of autologous serum eyedrops in the treatment of severe dry eye disease: a prospective randomized case-control study. Am J Ophthalmol 2005;139(2):242-246. [PubMed: 15733983]

28. Noble BA, Loh RS, MacLennan S, et al. Comparison of autologous serum eye drops with conventional therapy in a randomised controlled crossover trial for ocular surface disease. $\mathrm{Br} \mathrm{J}$ Ophthalmol 2004;88(5):647-652. [PubMed: 15090417]

** 29. Alio JL, Rodriguez AE, Ferreira-Oliveira R, Wrobel-Dudzinska D, Abdelghany AA. Treatment of Dry Eye Disease with Autologous Platelet-Rich Plasma: A Prospective, Interventional, NonRandomized Study. Ophthalmol Ther 2017;6(2):285-293. [PubMed: 28791607] Large consecutive case series of 368 patients with moderate to severe dry eye disease treated with autologous platelet-rich plasma drops as monotherapy. The majority of patients experienced improvement in dry eye symptoms and corneal flourescein staining with significant improvement in both OSDI and corneal fluorescein staining.

30. Partal A, Scott E. Low-cost protocol for the production of autologous serum eye drops by blood collection and processing centres for the treatment of ocular surface diseases. Transfus Med 2011;21(4):271-277. [PubMed: 21371142]

* 31. Toyos R, McGill W, Briscoe D. Intense pulsed light treatment for dry eye disease due to meibomian gland dysfunction; a 3-year retrospective study. Photomed Laser Surg 2015;33(1):4146. [PubMed: 25594770] Described the technique for IPL application with gland expression for treatment of dry eye disease secondary to meibomian gland dysfunction. Retrospective study of 91 patients found that the majority experienced satisfaction and majority had improved tear breakup time as judged by physician

32. Toyos R, Buffa C, Youngerman S. Dry-eye symptoms improve with intense pulsed light treatment. In. EyeWorld News Magazine2005.

33. Gupta PK, Vora GK, Matossian C, Kim M, Stinnett S. Outcomes of intense pulsed light therapy for treatment of evaporative dry eye disease. Can J Ophthalmol 2016;51(4):249-253. [PubMed: 27521662]

34. Vegunta S, Patel D, Shen JF. Combination Therapy of Intense Pulsed Light Therapy and Meibomian Gland Expression (IPL/MGX) Can Improve Dry Eye Symptoms and Meibomian Gland Function in Patients With Refractory Dry Eye: A Retrospective Analysis. Cornea 2016;35(3):318-322. [PubMed: 26785301]

35. Craig JP, Chen YH, Turnbull PR. Prospective trial of intense pulsed light for the treatment of meibomian gland dysfunction. Invest Ophthalmol Vis Sci 2015;56(3):1965-1970. [PubMed: 25678687]

* 36. Dell SJ, Gaster RN, Barbarino SC, Cunningham DN. Prospective evaluation of intense pulsed light and meibomian gland expression efficacy on relieving signs and symptoms of dry eye disease due to meibomian gland dysfunction. Clin Ophthalmol 2017;11:817-827. [PubMed: 28496300] Prospective study of patients who underewent four sequential sessions of IPL/MGX

Curr Opin Ophthalmol. Author manuscript; available in PMC 2020 May 01. 
which demonstrated signficant improvement in tear film breakup time, SPEED scores, meibomian gland scores, and corneal fluorescein staining scores as well as tear film osmolarity.

37. Fitzpatrick TB. The validity and practicality of sun-reactive skin types I through VI. Arch Dermatol 1988;124(6):869-871. [PubMed: 3377516]

38. Vora GK, Gupta PK. Intense pulsed light therapy for the treatment of evaporative dry eye disease. Curr Opin Ophthalmol 2015;26(4):314-318. [PubMed: 26058031]

39. Lane SS, DuBiner HB, Epstein RJ, et al. A new system, the LipiFlow, for the treatment of meibomian gland dysfunction. Cornea 2012;31(4):396-404. [PubMed: 22222996]

40. Finis D, Hayajneh J, Konig C, Borrelli M, Schrader S, Geerling G. Evaluation of an automated thermodynamic treatment (LipiFlow(R)) system for meibomian gland dysfunction: a prospective, randomized, observer-masked trial. Ocul Surf 2014;12(2):146-154. [PubMed: 24725326]

41. Finis D, Konig C, Hayajneh J, Borrelli M, Schrader S, Geerling G. Six-month effects of a thermodynamic treatment for MGD and implications of meibomian gland atrophy. Cornea 2014;33(12):1265-1270. [PubMed: 25321941]

42. Greiner JV. A single LipiFlow(R) Thermal Pulsation System treatment improves meibomian gland function and reduces dry eye symptoms for 9 months. Curr Eye Res 2012;37(4):272-278. [PubMed: 22324772]

* 43. Greiner JV. Long-Term (3 Year) Effects of a Single Thermal Pulsation System Treatment on Meibomian Gland Function and Dry Eye Symptoms. Eye Contact Lens 2016;42(2):99-107. [PubMed: 26222095] Prospective cohort study reporting outcomes over three years following a single thermal pulsation system treatment. Significant improvements in meibomian gland secretion scores and SPEED scores were sustained over this long-term follow-up period

44. Kim MJ, Stinnett SS, Gupta PK. Effect of thermal pulsation treatment on tear film parameters in dry eye disease patients. Clin Ophthalmol 2017;11:883-886. [PubMed: 28533678]

** 45. Hagen KB, Bedi R, Blackie CA, Christenson-Akagi KJ. Comparison of a single-dose vectored thermal pulsation procedure with a 3-month course of daily oral doxycycline for moderate-tosevere meibomian gland dysfunction. Clin Ophthalmol 2018;12:161-168. [PubMed: 29398903] Prospective, randomized parallel-group study of patients receiving either a single VTP treatment or three months of oral doxycycline for treatment of severe MGD. VTP was significantly more effective in improving dry eye symptoms and at least as effective at improving signs of MGD while avoiding side effects of long term doxycycline use

** 46. Blackie CA, Coleman CA, Holland EJ. The sustained effect (12 months) of a single-dose vectored thermal pulsation procedure for meibomian gland dysfunction and evaporative dry eye. Clin Ophthalmol 2016;10:1385-1396. [PubMed: 27555745] Prospective, cross-over study demonstrating that a single VTP treatment may provide significantly greater improvement in meibomian gland function and dry eye symptoms as compared to conventional treatments

47. Maskin SL. Intraductal meibomian gland probing relieves symptoms of obstructive meibomian gland dysfunction. Cornea 2010;29(10):1145-1152. [PubMed: 20622668]

48. Maskin SL, Testa WR. Growth of meibomian gland tissue after intraductal meibomian gland probing in patients with obstructive meibomian gland dysfunction. Br J Ophthalmol 2018;102(1): 59-68. [PubMed: 28592418]

49. Incekalan TK, Harbiyeli II, Yagmur M, Erdem E. Effectiveness of Intraductal Meibomian Gland Probing in Addition to the Conventional Treatment in Patients with Obstructive Meibomian Gland Dysfunction. Ocul Immunol Inflamm 2018:1-7.

50. Fermon S, Zaga IH, Alvarez Melloni D. [Intraductal meibomian gland probing for the treatment of blepharitis]. Arch Soc Esp Oftalmol 2015;90(2):76-80. [PubMed: 25001110]

51. Ma X, Lu Y. Efficacy of Intraductal Meibomian Gland Probing on Tear Function in Patients With Obstructive Meibomian Gland Dysfunction. Cornea 2016;35(6):725-730. [PubMed: 26967110]

52. Nakayama N, Kawashima M, Kaido M, Arita R, Tsubota K. Analysis of Meibum Before and After Intraductal Meibomian Gland Probing in Eyes With Obstructive Meibomian Gland Dysfunction. Cornea 2015;34(10):1206-1208. [PubMed: 26226474]

53. Sik Sarman Z, Cucen B, Yuksel N, Cengiz A, Caglar Y. Effectiveness of Intraductal Meibomian Gland Probing for Obstructive Meibomian Gland Dysfunction. Cornea 2016;35(6):721-724. [PubMed: 27032021] 
54. Wladis EJ. Intraductal meibomian gland probing in the management of ocular rosacea. Ophthalmic Plast Reconstr Surg 2012;28(6):416-418. [PubMed: 22836802]

* 55. Syed ZA, Sutula FC. Dynamic Intraductal Meibomian Probing: A Modified Approach to the Treatment of Obstructive Meibomian Gland Dysfunction. Ophthalmic Plast Reconstr Surg 2017;33(4):307-309. [PubMed: 28221295] Description of a novel technique for intraductal meibomian gland probing utilizing an operating microscope and von Grafe fixation forceps to assist in expression of meibum during probing. The authors report $91.4 \%$ of patients experience immediate symptomatic improvement in this retrospective study of 70 patients who underwent the procedure

56. Kislan T. Mibo Medical Group. 2018; http://mibomedicalgroup.com/articles.html. Accessed January 8, 2019.

57. Kenrick CJ, Alloo SS. The Limitation of Applying Heat to the External Lid Surface: A Case of Recalcitrant Meibomian Gland Dysfunction. Case Rep Ophthalmol 2017;8(1):7-12. [PubMed: 28203190]

58. Borchman D, Foulks GN, Yappert MC, et al. Human meibum lipid conformation and thermodynamic changes with meibomian-gland dysfunction. Invest Ophthalmol Vis Sci 2011;52(6):3805-3817. [PubMed: 21398284]

59. Connor CG, Choat C, Narayanan S, Kyser K, Rosenberg B, Mulder D. Clinical Effectiveness of Lid Debridement with BlephEx Treatment. Investigative Ophthalmology \& Visual Science. 2015;56(7):4440-4440.

60. Connor CG, Narayanan S, Miller W. Reduction in inflammatory marker matrix metalloproteinase-9 following lid debridement with BlephEx. Investigative Ophthalmology \& Visual Science. 2017;58(8):498-498.

61. Badawi D. A novel system, TearCare $((\mathrm{R}))$, for the treatment of the signs and symptoms of dry eye disease. Clin Ophthalmol 2018;12:683-694. [PubMed: 29692600]

** 62. Dieckmann G, Fregni F, Hamrah P. Neurostimulation in dry eye disease-past, present, and future. Ocul Surf 2018. Review of neuromodulatory therapies in ocular and non-ocular diseases that includes description of the lacrimal function unit; also includes review of recent studies on intranasal tear neurostimulation for dry eye disease

63. Gumus K, Pflugfelder SC. Intranasal Tear Neurostimulation: An Emerging Concept in the Treatment of Dry Eye. Int Ophthalmol Clin 2017;57(2):101-108. [PubMed: 28282317]

64. Friedman NJ, Butron K, Robledo N, Loudin J, Baba SN, Chayet A. A nonrandomized, open-label study to evaluate the effect of nasal stimulation on tear production in subjects with dry eye disease. Clin Ophthalmol 2016;10:795-804. [PubMed: 27217719]

65. Gumus K, Schuetzle KL, Pflugfelder SC. Randomized Controlled Crossover Trial Comparing the Impact of Sham or Intranasal Tear Neurostimulation on Conjunctival Goblet Cell Degranulation. Am J Ophthalmol 2017;177:159-168. [PubMed: 28302532]

* 66. Sheppard JD, Torkildsen GL, Geffin JA, et al. Characterization of tear production in subjects with dry eye disease during intranasal tear neurostimulation: Results from two pivotal clinical trials. Ocul Surf 2018. Report of 1-day crossover and 180 day prospective cohort studies of intranasal tear neurostimulation demonstrating significantly higher Schirmer scores both immediately following a single use and after 180 days of consistent use of the device.

67. Romero-Rangel T, Stavrou P, Cotter J, Rosenthal P, Baltatzis S, Foster CS. Gas-permeable scleral contact lens therapy in ocular surface disease. Am J Ophthalmol 2000;130(1):25-32. [PubMed: 11004256]

68. Agranat JS, Kitos NR, Jacobs DS. Prosthetic replacement of the ocular surface ecosystem: impact at 5 years. Br J Ophthalmol 2016;100(9):1171-1175. [PubMed: 26644423]

69. Cressey A, Jacobs DS, Remington C, Carrasquillo KG. Improvement of chronic corneal opacity in ocular surface disease with prosthetic replacement of the ocular surface ecosystem (PROSE) treatment. Am J Ophthalmol Case Rep 2018;10:108-113. [PubMed: 29552666]

70. Dimit R, Gire A, Pflugfelder SC, Bergmanson JP. Patient ocular conditions and clinical outcomes using a PROSE scleral device. Cont Lens Anterior Eye. 2013;36(4):159-163. [PubMed: 23499361] 
71. Lim P, Ridges R, Jacobs DS, Rosenthal P. Treatment of persistent corneal epithelial defect with overnight wear of a prosthetic device for the ocular surface. Am J Ophthalmol 2013;156(6):10951101. [PubMed: 24075432]

72. Theophanous C, Irvine JA, Parker P, Chiu GB. Use of Prosthetic Replacement of the Ocular Surface Ecosystem Scleral Lenses in Patients with Ocular Chronic Graft-versus-Host Disease. Biol Blood Marrow Transplant. 2015;21(12):2180-2184. [PubMed: 26234721]

* 73. DeLoss KS, Le HG, Gire A, Chiu GB, Jacobs DS, Carrasquillo KG. PROSE Treatment for Ocular Chronic Graft-Versus-Host Disease as a Clinical Network Expands. Eye Contact Lens 2016;42(4):262-266. [PubMed: 26448446] Report of a large cohort of patients treated with PROSE for ocular cGVHD, demonstraing significant improvemetn in NEI VFQ scores following treatment

74. Kloek CE, Jeng-Miller KW, Jacobs DS, Dunn IF. Prosthetic Replacement of the Ocular Surface Ecosystem Treatment of Ocular Surface Disease After Skull Base Tumor Resection. World NeuroSurg 2018;110:e124-e128. [PubMed: 29107724]

* 75. Papakostas TD, Le HG, Chodosh J, Jacobs DS. Prosthetic replacement of the ocular surface ecosystem as treatment for ocular surface disease in patients with a history of Stevens-Johnson syndrome/toxic epidermal necrolysis. Ophthalmology. 2015;122(2):248-253. [PubMed: 25282251] Median visual acuity and visual function of patients treated with PROSE for ocular surface disease due to SJS/TEN both improved significantly in this large cohort of 86 patients

76. Stason WB, Razavi M, Jacobs DS, et al. Clinical benefits of the Boston Ocular Surface Prosthesis. Am J Ophthalmol 2010;149(1):54-61. [PubMed: 19878920]

* 77. Nguyen MTB, Thakrar V, Chan CC. EyePrintPRO therapeutic scleral contact lens: indications and outcomes. Can J Ophthalmol 2018;53(1):66-70. [PubMed: 29426444]

* 78. Cheng AM, Zhao D, Chen R, et al. Accelerated Restoration of Ocular Surface Health in Dry Eye Disease by Self-Retained Cryopreserved Amniotic Membrane. Ocul Surf 2016;14(1):56-63. [PubMed: 26387870]

* 79. McDonald MB, Sheha H, Tighe S, et al. Treatment outcomes in the DRy Eye Amniotic Membrane (DREAM) study. Clin Ophthalmol 2018;12:677-681. [PubMed: 29670328] Cryopreserved amniotic membrane treatment for 5 days improved the ocular surface and overall DEWS score for $88 \%$ of patients with with severe, refractory dry eye disease

80. Vijmasi T, Chen FY, Balasubbu S, et al. Topical administration of lacritin is a novel therapy for aqueous-deficient dry eye disease. Invest Ophthalmol Vis Sci 2014;55(8):5401-5409. [PubMed: 25034600]

81. McNamara NA, Ge S, Lee SM, et al. Reduced Levels of Tear Lacritin Are Associated With Corneal Neuropathy in Patients With the Ocular Component of Sjogren's Syndrome. Invest Ophthalmol Vis Sci 2016;57(13):5237-5243. [PubMed: 27711909]

82. Schmidt TA, Sullivan DA, Knop E, et al. Transcription, translation, and function of lubricin, a boundary lubricant, at the ocular surface. JAMA Ophthalmol 2013;131(6):766-776. [PubMed: 23599181]

* 83. Lambiase A, Sullivan BD, Schmidt TA, et al. A Two-Week, Randomized, Double-masked Study to Evaluate Safety and Efficacy of Lubricin $(150 \mathrm{mug} / \mathrm{mL})$ Eye Drops Versus Sodium Hyaluronate (HA) 0.18\% Eye Drops (Vismed(R)) in Patients with Moderate Dry Eye Disease. Ocul Surf 2017;15(1):77-87. [PubMed: 27614318] This trial compared outcomes following two weeks of Lubricin use as compared to sodium hyaluronate and found that Lubricin significantly outperformed HA across multiple endpoints for both ocular symptoms and signs of disease

84. Sosne G, Dunn SP, Kim C. Thymosin beta4 significantly improves signs and symptoms of severe dry eye in a phase 2 randomized trial. Cornea 2015;34(5):491-496. [PubMed: 25826322]

* 85. Sosne G, Kleinman HK. Primary Mechanisms of Thymosin $\beta 4$ Repair Activity in Dry Eye Disorders and Other Tissue Injuries. Investigative Ophthalmology \& Visual Science. 2015;56(9): 5110-5117. [PubMed: 26241398] Comprehensive review of the mechanism of action of Thymosin $\beta 4$ in treating ocular surface disease, as well as preclinical and early clinical trial results.

* 86. Dunn SP, Heidemann DG, Chow CY, et al. Treatment of chronic nonhealing neurotrophic corneal epithelial defects with thymosin beta 4. Arch Ophthalmol 2010;128(5):636-638. [PubMed: 20457990] 
87. Sosne G, Szliter EA, Barrett R, Kernacki KA, Kleinman H, Hazlett LD. Thymosin beta 4 promotes corneal wound healing and decreases inflammation in vivo following alkali injury. Exp Eye Res 2002;74(2):293-299. [PubMed: 11950239]

88. Kim CE, Kleinman HK, Sosne G, et al. RGN-259 (thymosin beta4) improves clinically important dry eye efficacies in comparison with prescription drugs in a dry eye model. Sci Rep 2018;8(1): 10500. [PubMed: 30002412]

* 89. Murri MS, Moshirfar M, Birdsong OC, Ronquillo YC, Ding Y, Hoopes PC. Amniotic membrane extract and eye drops: a review of literature and clinical application. Clin Ophthalmol 2018;12:1105-1112. [PubMed: 29950805] Comprehensive review of the production, clinical applications, ongoing studies, benefits and challenges of amniotic membrane extract eye drops.

90. Baudouin C, Figueiredo FC, Messmer EM, et al. A randomized study of the efficacy and safety of $0.1 \%$ cyclosporine A cationic emulsion in treatment of moderate to severe dry eye. Eur J Ophthalmol 2017;27(5):520-530. [PubMed: 28362054]

91. Ebihara N, Ohashi Y, Uchio E, et al. A large prospective observational study of novel cyclosporine $0.1 \%$ aqueous ophthalmic solution in the treatment of severe allergic conjunctivitis. J Ocul Pharmacol Ther 2009;25(4):365-372. [PubMed: 19441889]

92. Leonardi A, Van Setten G, Amrane M, et al. Efficacy and safety of $0.1 \%$ cyclosporine A cationic emulsion in the treatment of severe dry eye disease: a multicenter randomized trial. Eur $\mathbf{J}$ Ophthalmol 2016;26(4):287-296. [PubMed: 27055414]

93. Quintana-Hau JD, Garcia-Gallegos VM, López-Sánchez I, Mondragón-Flores R, HernándezSantoyo A, Cuevas-Pacheco G. Ultra Structural Characterization of a New Cyclosporin-A Aqueous Solution for Ophthalmic Application (MODUSIK-A OftenoTM). Investigative Ophthalmology \& Visual Science. 2003;44(13):3461-3461.

94. Tauber J, Schechter BA, Bacharach J, et al. A Phase II/III, randomized, double-masked, vehiclecontrolled, dose-ranging study of the safety and efficacy of OTX-101 in the treatment of dry eye disease. Clin Ophthalmol 2018;12:1921-1929. [PubMed: 30323548]

95. Torkildsen G, Wirta D, Lonsdale J, et al. A Clinical Phase 2 Study to Assess Safety, Efficacy, and Tolerability of CyclASol® for the Treatment of Dry Eye Disease. 2017.

96. Lindstrom RL, Kaufman HE, Skelnik DL, et al. Optisol corneal storage medium. Am J Ophthalmol 1992;114(3):345-356. [PubMed: 1524127]

Curr Opin Ophthalmol. Author manuscript; available in PMC 2020 May 01. 


\section{Key Points}

- Recently approved topical lifitegrast shows promise in improving dry eye symptoms at least as well as topical cyclosporine, with a faster timeline for efficacy

- Blood products and amniotic membranes replace beneficial growth factors and cytokines, speeding corneal healing while decreasing inflammation on the ocular surface

- A variety of in-office procedures may improve meibomian gland function, primarily by heating and encouraging expression of viscous meibum, though many lack large-scale prospective studies

- The intranasal tear neurostimulator represents the first approved device to increase tear production in patients with dry eye disease

- $\quad$ Ongoing studies for a number of medications that contain naturally occurring glycoproteins hold promise for future treatments, while other medications seek to optimize the success of topical cyclosporine via reformulations at higher concentrations or in compound with chondroitin sulfate

\section{Synopsis}

In this article, we review advances in the treatment of dry eye disease. We will discuss new therapeutic agents including topical lifitegrast and platelet rich plasma drops. In addition, we will review advances in treatments for meibomian gland dysfunction (MGD) and blepharitis such as intense pulsed light (IPL), the LipiFlowTM system, meibomian gland probing, MiBo Thermoflo ${ }^{\mathrm{TM}}$, Blephex ${ }^{\mathrm{TM}}$, and the TearCare ${ }^{\circledR}$ System. A new device, the intranasal tear neurostimulator, will also be discussed, as well as specialty contact lenses that can be used for the treatment of ocular surface disease such as Prokera $^{\mathrm{TM}}$, the PROSE lens, and EyePrintPRO ${ }^{\mathrm{TM}}$. Finally we will discuss new medications that are in development. 


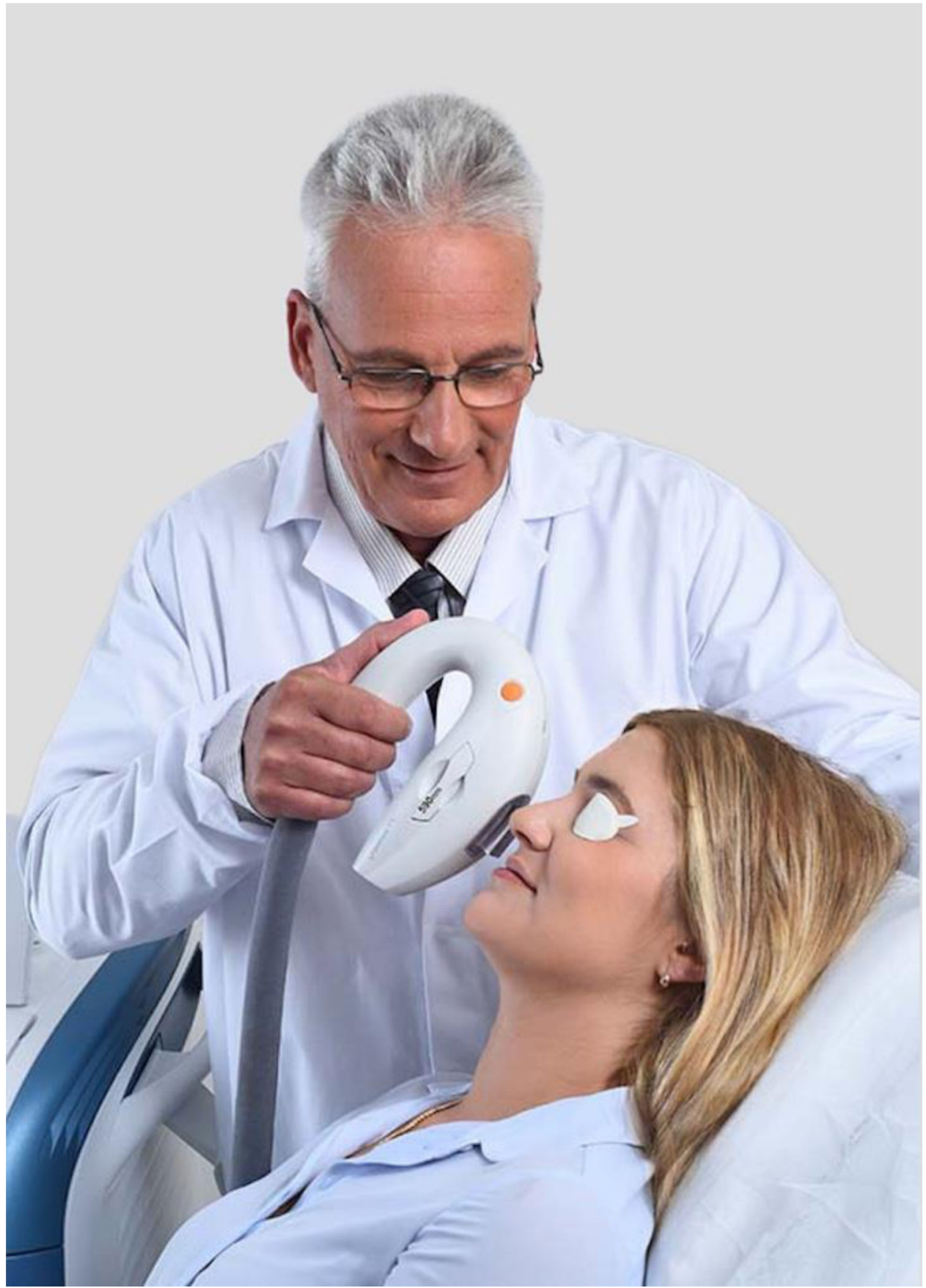

Figure 1:

Application of Intense pulsed light (IPL) 


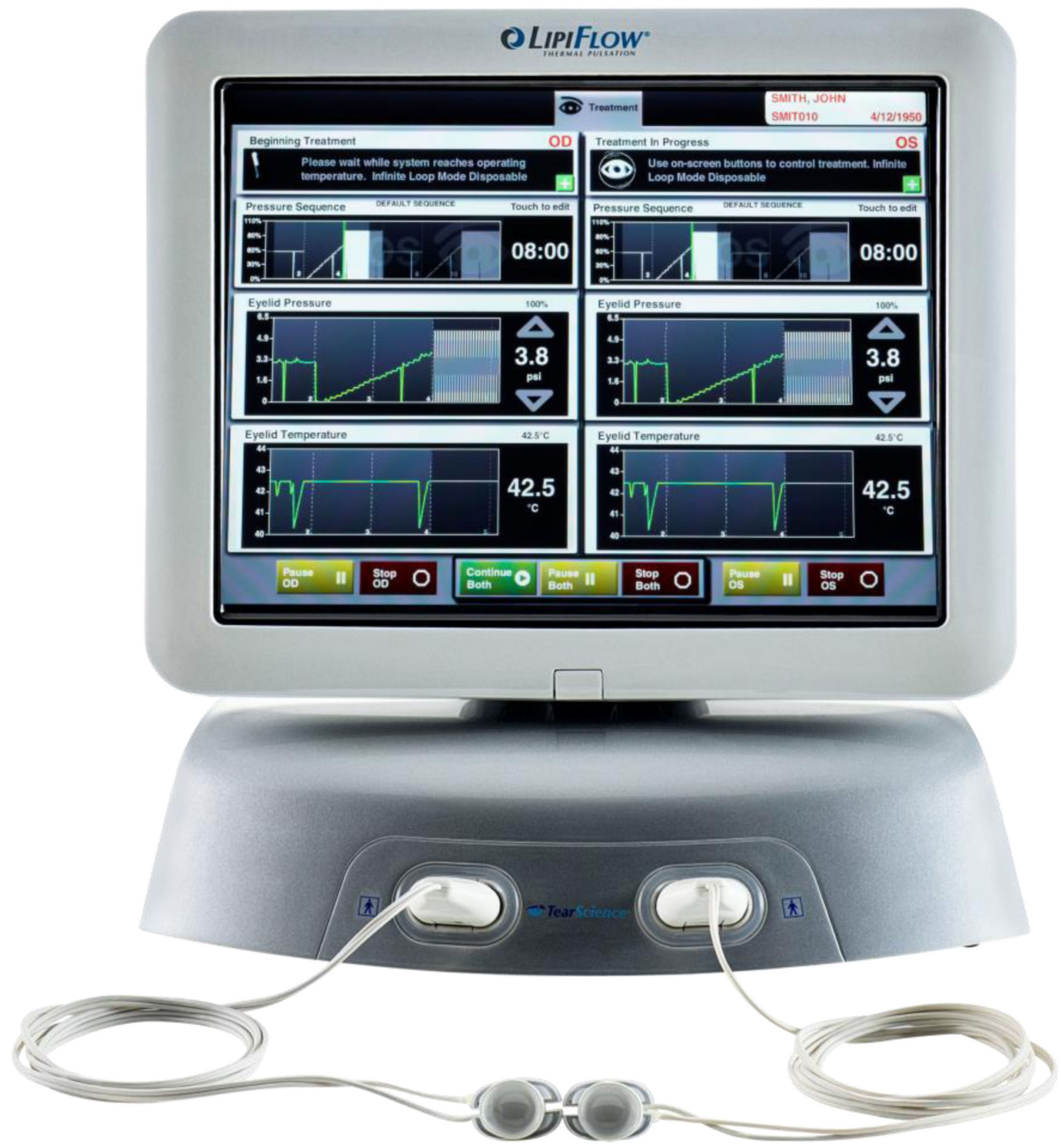

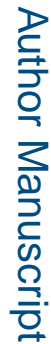

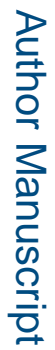

Figure 2:

Thermal pulsation system 

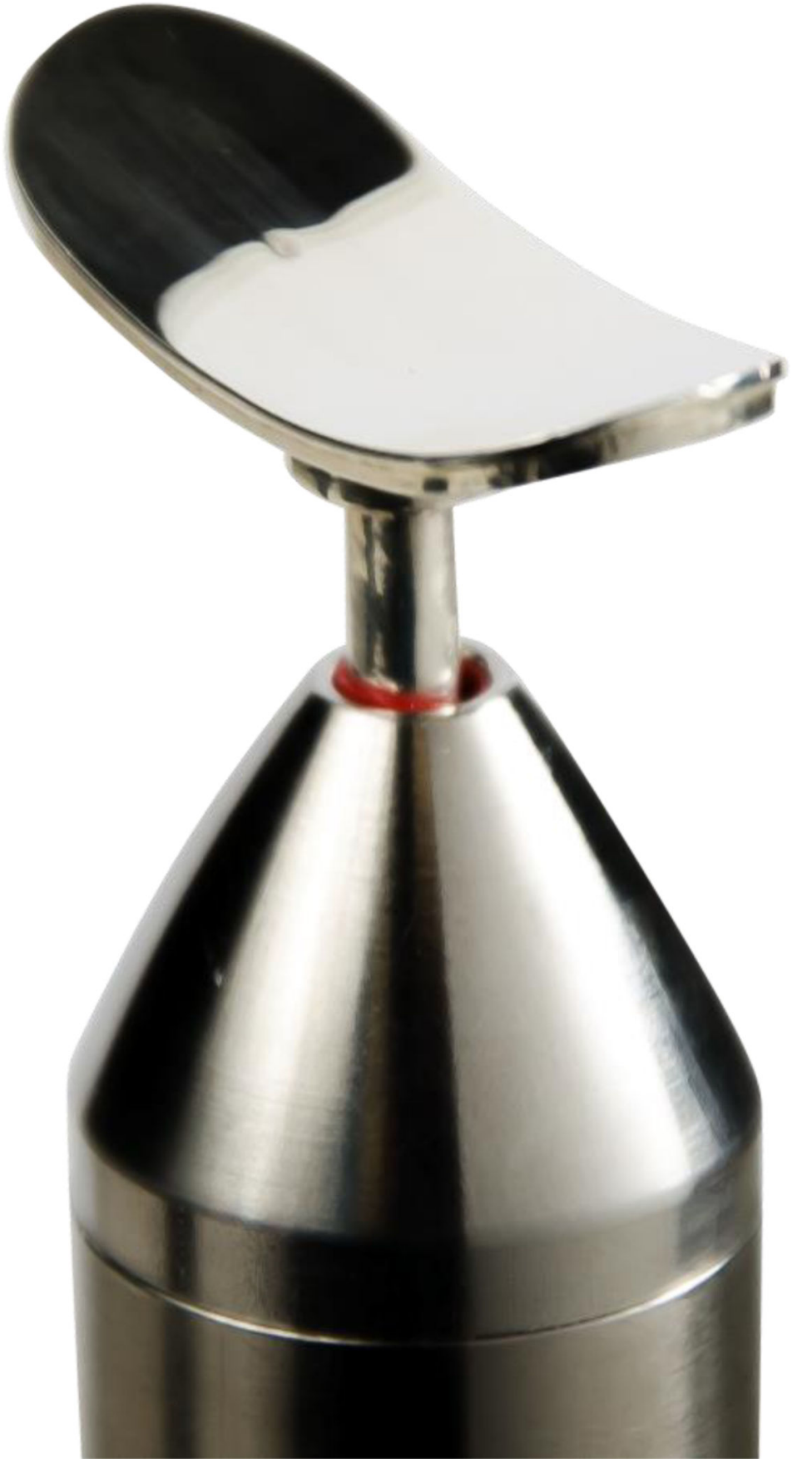

Figure 3:

MiBo ThermoFlo application device 


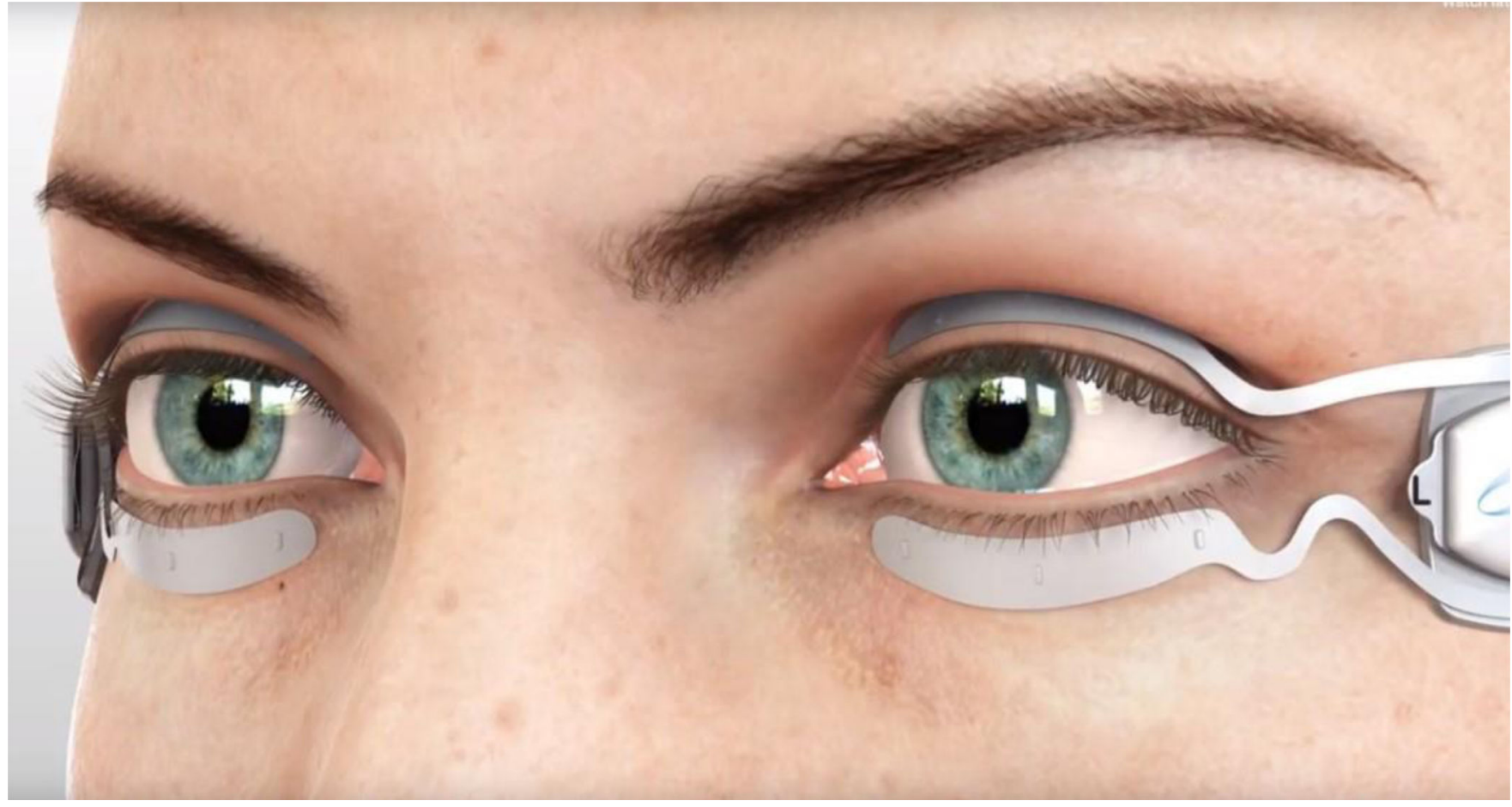

Figure 4:

TearCare iLid device 


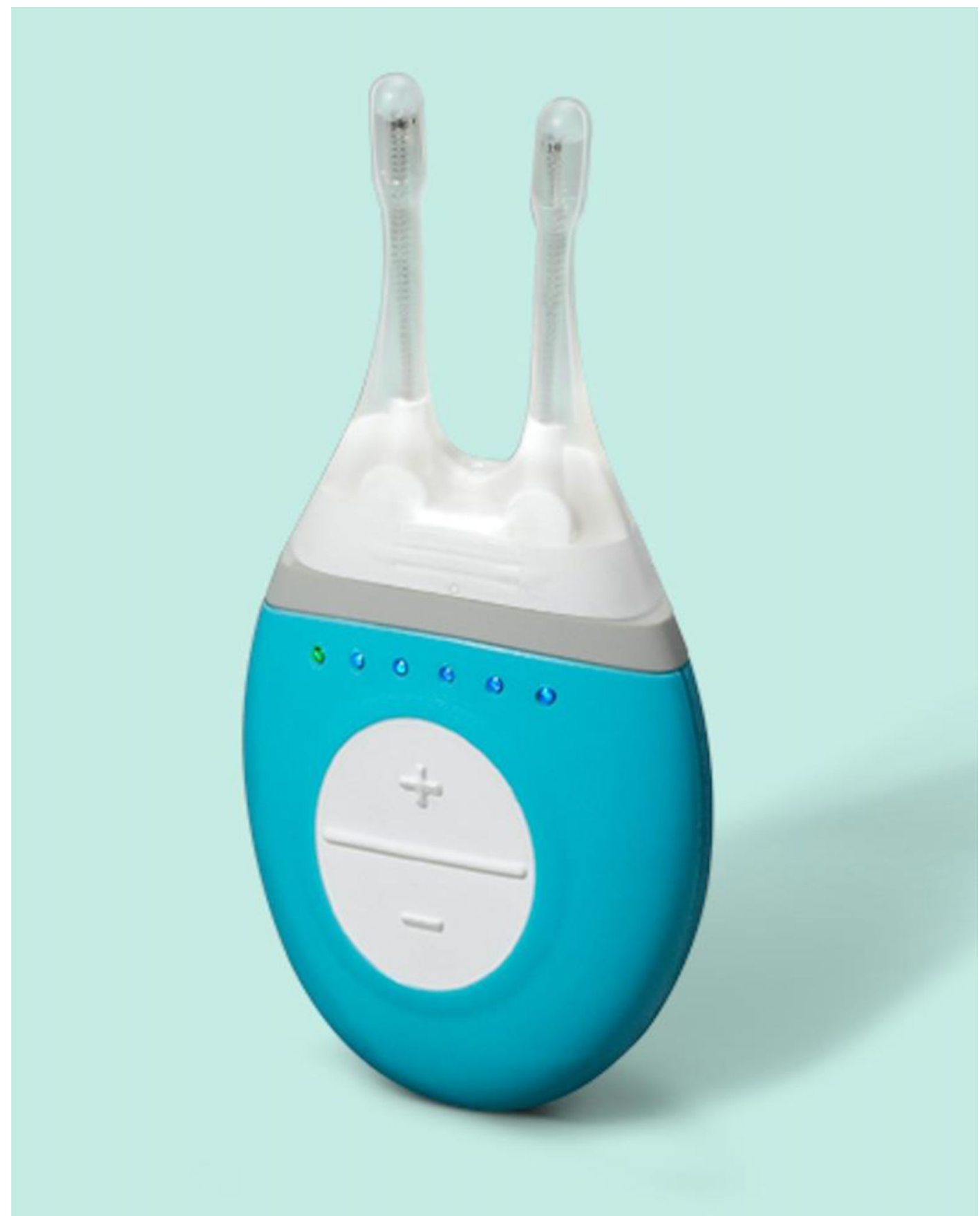

Figure 5:

Intranasal Tear Neurostimulator 